\title{
Identifying and Prioritizing the Research Needs Related to Mental Health in Gaza Strip, Palestine
}

\author{
Nasser Ibrahim Abu-El-Noor, Yousef Ibrahim Aljeesh \\ College of Nursing, Islamic University of Gaza, Gaza, Palestine \\ Email: naselnoor@iugaza.edu.ps, yjeesh@iugaza.edu.ps
}

Received 24 September 2014; revised 20 October 2014; accepted 18 November 2014

Academic Editor: Gjumrakch Aliev, University of Atlanta, USA

Copyright (C) 2015 by authors and Scientific Research Publishing Inc.

This work is licensed under the Creative Commons Attribution International License (CC BY). http://creativecommons.org/licenses/by/4.0/

(c) (i) Open Access

\begin{abstract}
Background: An important function of research is to identify community needs in certain fields. As mental health is a vital issue to us, identifying and prioritizing mental health needs is important to policy makers to help them in setting goals for different programs that meet the needs of a specific community in a certain health area. Purpose: To identify the health research needs in the field of mental health in Gaza Strip, Palestine. Design and Methods: The design was a qualitative design using focus group, need assessment and non-structured interview for data collection. Participants: Participants included three focus groups. First group consisted of students enrolled in a master program of community mental health nursing; second group included seven faculty members who were specialists in mental health; and third group included six key persons from the ministry of health and the chair of community mental health program in WHO at Gaza Strip. Results: Results of this qualitative study revealed several themes that emerged from the thematic analysis. Many health research needs were identified by participants. These needs included the following topics: stigma, family integration, aggression of children born to substance-abuse fathers, post-partum depression, counseling, talking therapy in clinical practice, behavioral problems, tramadole abuse, risk factors leading to drug dependence, autism, exploring the role of religion in mental health, and Wellness Recovery Action Plan. Implication for Practice: Well designed studies will help to identify and prioritize the health needs for a specific community. Identifying the needs related to community mental health is one of the first steps to help in pushing these needs into the agenda of health policy makers, who then will work to set goals and design policies and programs that aiming to meet the needs of the community, which hopefully will produce a community with less mental health problems.
\end{abstract}

\section{Keywords}

Research Needs, Research Priorities, Mental Health, Focus Group, Gaza Strip 


\section{Introduction}

The new millennium has brought many challenges and opportunities for health advancement. This is especially true given the growing number of adverse incidence rates, morbidity, and mortality for different diseases and health problems, including mental health. Scientific research provides new insights for investigating these challenges [1].

Scientific research is defined as "the systematic, controlled, empirical and critical investigation of hypothetical propositions about presumed relations among natural phenomena” ([2], p. 41). The function of scientific research includes producing explanations, predictions and solutions for people's problems. Also, scientific research adds facts and theories to the body of overall scientific knowledge [2]. Health research also seeks to analyze the objectives and functions of health services including the social, political, and economic factors that impact efficiency, effectiveness, and priorities of these services [3]. On the other hand, Israel et al. [4] added that other benefits of research included ensuring that research topics reflected the major concerns of local communities and improving the well-being and health of the nation. In fact, research related to health services became more prominent in recent years because of the increased demand for effectiveness of care, development of guidelines and standards, and for setting an agenda for health services [5].

Setting a national research agenda requires involvement from a broad array of stakeholders, including researchers, health care professionals, consumers of health care services, and others. Participation of these diverse groups is essential to set priorities of health research agendas that advance our understanding of relevant health issues and help set goals for national health. Researchers must be proactive in predicting different health needs and seeking solutions to relevant health problems, including mental health, which is congruent with community concerns [6] [7].

The field of mental health is very dynamic. The number of people who suffer from mental disorders is on the increase accounting for $14 \%$ of the disease burden worldwide [8]. For example, substance abuse, by itself, accounts for $4 \%$ of the total burden [9]. It is also expected that depression will be, by the year of 2020, the second leading cause of disease burden [10].

Unfortunately, data about the prevalence of mental disorders in Gaza Strip are not available. With the dynamicity in the field of mental health, it would be imprudent to use the results of yesterday's research to set the agenda for today's practice. Therefore, it is imperative that scientific research in the field of mental health to be updated to adapt to dynamic changes in the field. Current research is also needed to predict the needs of and seek solutions for relevant mental health problems and to help pave the road for changes in policies and laws, especially, those that control who, how much and where people would receive care [11] [12]. In fact, research in the field of mental health was, uncoordinated, ad hoc, and often undertaken by different agencies with no centralization and did not consider priorities in the mental health sector [13] [14]. Also, professionals in mental health had not emphasized or prioritized research in the field of mental health as an integral part in the practice of mental health [15] [16]. Thus, research in the field of mental health remains sporadic and results are rarely reported in the literature [17]. As a result, solutions to various mental health disorders are usually not generated through the research [18].

As it is almost impossible to fully understand and anticipate how mental healthcare will be restructured over the next 20 years [19], researchers aim to identify and prioritize research topics of importance in the field of mental health in Gaza Strip, Palestine.

\section{Methodology of the Study}

\subsection{Design}

A qualitative design was used in this study. Qualitative research provides the basis by which one can gain an in-depth understanding of how the researchers and policy makers identify and prioritize the needs of research studies related to mental health [20].

\subsection{Participants and Settings}

In this study, we used a non-probability convenience sample-focus group interview. This sample is commonly used in qualitative research to enhance selection of participants who are able to give information more specific to research questions. Purposive sampling allows for identifying participants who meet predetermined criteria 
useful for the purpose of the study [20]. The sample consisted of nine participants who were selected from five different organizations that have interest in mental health research. Participants came from Faculty of Nursing at Islamic University of Gaza (IUG), Psychology Department at IUG, Ministry of Health in Gaza (MoH), Human Resources Department at the MoH and the World Health Organization (WHO) office in Gaza. The data were collected by performing in-depth interviews. The purpose of these interviews was to obtain rich information to help identify and prioritize the needs for research studies related to mental health disorders in Gaza Strip. The interview questions were designed to be flexible, understandable, and open to the extent of participants' experiences with mental health. Each participant gave rich information, because they spoke about their own experiences. A qualitative research supposes that reality is constructed from the meaning that each participant takes from their experience [21].

\subsection{Analysis}

Analysis of qualitative data were carried out by using three elements namely: concepts, categories and propositions. Concepts are the basic unit of analysis since it comes from conceptualization of data and not the actual data itself. Categories are higher in level and more abstract than the concepts. Propositions help to connect the relationship between the categories and concepts [22]. It is important for the researcher of qualitative research to capture his/her subjective experiences. Therefore, the interviews and the subsequent data analysis, allowed the researchers to develop an understanding of that reality in the sense of participant's belief and view [21]. In this study context, the transcribed interview files were read several times. This helped the researchers to ensure that interviews were transcribed correctly.

In content comparative analysis, data were systematically analyzed within specific steps. Raw data provided by the participants were carefully read, word by word and line by line. Codes were developed through a process of open coding. Coding in qualitative research is a word or a string of words used as titles for categories generated during analysis process. Coding is the first step in moving beyond concrete statements in the data in order to make analytical interpretations [22]. Qualitative methods use codes to categorize data rather than to quantify it. This method can help in generating subcategories and themes from the textual data [20]. The descriptive codes, followed with further analysis, yielded categories representing the themes that emerged from the data. The data were compared for similarities and differences; in order to give meaningful, interrelated categories and subcategories. By using content comparative analysis, the researchers compared each interview with the others [20]. The researchers also worked to obtain multiple view points of the phenomenon by interviewing individuals from different organizations in Gaza Strip. Finally, the researchers actively utilized these techniques for the purpose of developing objectivity. Through this process, researchers' own subcategories about phenomena were explored, leading to new discoveries.

\section{Results}

After the researchers had carefully examined the scripts of the participants' responses, they identified the emerging themes and subthemes and coded them into the following major themes: surveillance, culture sensitivity, and intervention (Figure 1). Under each theme, there were several subthemes that emerged. Under the surveillance theme, the following subthemes emerged: drug abuse, suicidal rate, and risk factors for mental disorders. Under the culture sensitivity theme, the subthemes of stigma, culture misconception, socialization and psychopathology problems emerged. Finally, the mental health interventions theme included the following subthemes: Wellness Recovery Action Plan (WRAP), effectiveness and impact assessment of mental health and psychosocial interventions and standardization of Western imported interventions and research tools.

\subsection{Surveillance}

The majority of participants mentioned that there is a lack of surveillance of mental disorders in Gaza Strip. The $(\mathrm{MoH})$ does not have a database about the prevalence of different mental health disorders such as; drug abuse, suicide rate, autism, and community risk factors for mental disorders. One of the participants mentioned:

"We feel embarrassed when we have visitors from other countries. They ask about the prevalence of a certain psychological disorder. I don't know how to answer this question. Thus, I prefer to start with performing studies to assess the prevalence of the different psychotic disorders in Gaza Strip because these numbers are not available at the Ministry of Health.” 


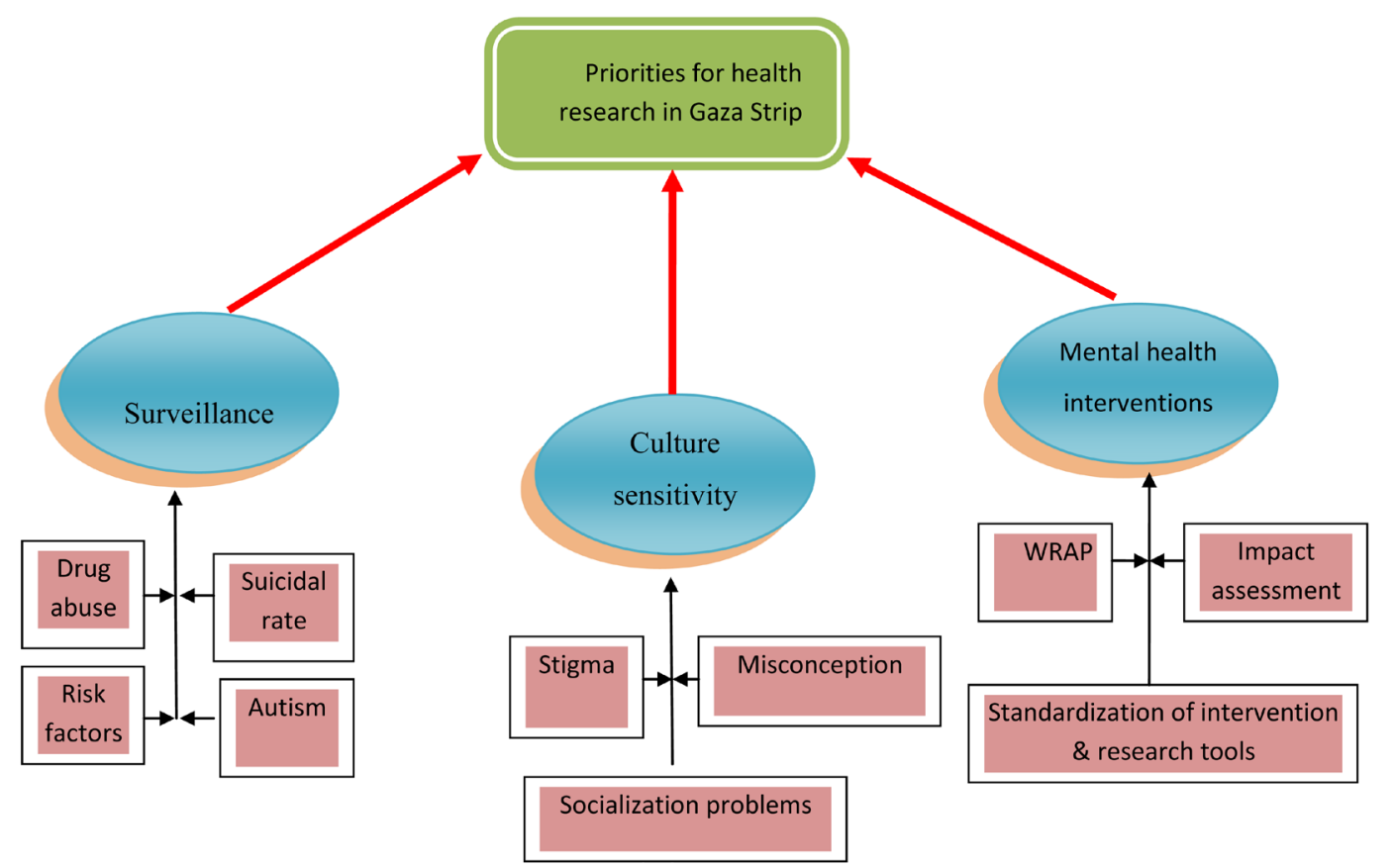

Figure 1. Priorities in mental health research in Gaza Strip, Palestine.

Participants mentioned that the lack of such data can hinder the research process as data availability helps them to know where to start and identify the priority areas in relation to research. It can also hinder the estimation of the sample size and hinder reaching potential population which will affect the generalization of the results. Therefore, participants suggested that one of the most important areas of research in mental health should start with surveillance.

"We need to know the prevalence rate for the most important mental disorders in Gaza Strip. This will help us a lot in conducting new studies. It will also help in knowing where to start and on what topics to focus on. It would be inefficient to conduct research on a topic that has low incidence and leaves other disorders that have much higher incidence rates.”

\subsection{Culture Sensitivity}

A great number of participants favored to start with the culture sensitivity and culture perception about mental health disorders. They mentioned that issues related to stigma and culture misconception about mental disorders are a priority for research in mental health disorders. Research in this area can help the public to accept the concept of mental disorders and therefore, help in the treatment of people who suffer from such disorders. Other participants indicated that starting with research related to interventions in mental disorders would be of no benefit if the community has a negative perception about mental health disorders. People here in Gaza Strip don’t accept mental illness and they would be ashamed if a family member suffered from a mental disorder. They would try to conceal the incidence and might not seek treatment.

"If we started with interventions, what would the benefit of implementing those interventions be, as some people won't accept the idea of psychological disorders and seek treatment? Therefore, starting with interventions might be useless. The stigma about psychological disorders is high in our community. I believe that we need to start assessing the level of stigma and how we can overcome this problem.”

The subthemes that emerged under this theme included stigma, culture misconception, and socialization and psychopathology problems.

\subsection{Mental Health Interventions}

Other participants preferred to start with research related to interventions. Examples of interventions mentioned 
in the discussion included: Wellness Recovery Action Plan (WRAP), behavioral and cognitive therapy, etc. Besides conducting research related to direct treatment interventions, others suggested conducting research studies measuring the effectiveness and the impact of assessment for mental health and psychological interventions. In order to conduct such studies, participants mentioned that there should be a standardization of tools and methods for intervention and research instruments (i.e. standards to use international tools and instruments such as autism scale, depression scale, etc.).

"I believe that the best start is to focus on interventions for psychological disorders because we need something practical to help this group of vulnerable people.

In implementing research in the area of mental health, I believe that we need to get well developed international instruments that have been already tested for validity and reliability. The lack of these instruments, I believe, is one of the barriers for conducting research in the area of mental health. I think that the best start for us is to contact international institutes and academic organizations that have good reputations in mental health research. Then we can translate those instruments into the Arabic language. I believe this will be a good start.”

\section{Discussion}

The result of this study identified the priority of research in the field of mental health aiming to reduce the burden of mental disorders in Gaza Strip, Palestine. Participants achieved a consensus about main themes. Three main themes were identified by the participants. These themes were surveillance, culture sensitivity to issue of mental disorders, and related interventions. If the results of this study were followed thoroughly, it could be formative in establishing baseline knowledge for future research in the field of mental health in Gaza Strip.

The results of this study are generally congruent with the results of other similar studies but do differ in parts. For example, participants of McAllister et al., [18] identified the following areas as priorities in mental health research: emotional well-being, service quality, accountability, family involvement and training and education of mental health professionals. Tomlinson et al. [23] reported that research in mental health policy was rated more important than research aiming to explore new interventions for mental disorders, such as drugs or technologies.

In another study, Gregorio et al. [24] identified several topics as a priority for mental health research in Brazil. The most important priorities were identification and treatment of common mental disorders, determining the cost-effectiveness of psychotropic drugs, policies related to controlling the consumption of alcohol and drugs at the population level, and identification of barriers to the access to treatment. Jorm et al. [25] reported that research on prevention and promotion, evaluation of mental health services, and psychological and social treatments were rated by different stakeholders as the most important topics for research in mental health.

Classifying interventions for mental health disorders was a common topic on the top lists of research in mental health for different studies, including our study. What is strange is that the participants of this study did not classify topics that were classified in other studies as important for mental health research. For example, despite that the population of Gaza Strip is subject to different mental health disorders due to the political situation in the area, our participants did not include research in the area of prevention of mental health disorders as a priority. Although that the majority of primary care doctors and nurses had not received official in-service training related to mental health in the last five years [26], our study participants did not include training or education of mental health professionals as a priority for research in the field of mental health.

Our participants rated the topic of surveillance as a priority for mental health research. This was not included in the literature as a priority. This could be related to the fact that the countries where these studies were conducted had an already established system for reporting mental health disorders and have the statistics available for health researchers. Surveillance is defined as "the ongoing systematic collection, consolidation and analysis of data and the dissemination of this information to those who need it so that action may be taken. It can be seen as the compass that guides disease control activities and measures the impact of the system” ([27], p. 13). Since surveillance would be of high relevance to researchers; this would be an important topic to be addressed in mental health research. According to WHO, Department of Vaccines and Biologicals ([27], p. 13), the "purposes of disease surveillance include:

- Predicting or detecting disease outbreaks with a view to investigation and containment;

- Identifying high-risk populations and areas requiring special attention; 
- Guiding disease eradication, elimination and control initiatives;

- Identifying areas where the system performs poorly so that corrective measures can be taken;

- Determining the burden of a disease on the health system and/or community in terms of incidence/prevalence or proportional morbidity/mortality."

Another issue that was mentioned as a high priority for research [18] [24] in mental health but was not addressed by the participants of this study is health policy related to mental health. In fact, there is absence of legislation and officially approved policy in the Palestinian health care system related to mental health [26].

The researchers believe that research in mental health should focus on prevention and devising interventions for existing mental disorders at primary, secondary, and tertiary levels. Evaluation of availability and feasibility of existing mental health services, polices related to mental health, interventions related to decrease incidence of drug abuse, investigating barriers to mental health care are topics that might need to be addressed by mental health research.

Finally, special attention should be paid to care for children and adolescents. The prevalence of mental health problems among children and adolescents was estimated to affect 10\% - 36\% of the children in the Eastern Mediterranean, which is significantly higher than the estimates among the same group in developed countries [28]. This would be important to the population of Palestine; knowing that children 15 years and younger constitute more than $40.8 \%$ of the population [29].

\section{Recommendations}

In order to enhance research in the field of mental health in Gaza Strip, Palestine, the authors make the following recommendations. First, the authors recommend that heath education programs for physicians, nurses, and other health care professionals focus on primary and secondary levels of mental health disorders.

Second, the authors believe that the media (TV, radio, newspaper, internet, etc.) can play a major role in minimizing culture sensitivity toward mental health disorders and help the public to accept people suffering from mental disorders. Therefore, the authors encourage mental health care professionals to become more involved in the media and participate in discussion and talk-shows. They can help educate the public as to the nature of mental health illness, its risk factors, their role toward the mentally ill, and how they can participate in their treatment. The authors believe that the more the public learns about mental health issues, the more they will accept it and the harder they will work to prevent it.

Third, the authors believe that involving consumers of health care services in setting priorities for research in the area of mental health is very crucial. Therefore, they recommend involving patients and their families in future studies. Finally, the authors would encourage health policy makers to pay more attention to mental health disorders and give it the same level of attention as physical disorders.

\section{References}

[1] Litt, J., Tran, N., Malecki, K.C., Neff, R., Resnick, B. and Burke, T. (2004) Identifying Priority Health Conditions, Environmental Data, and Infrastructure Needs: A Synopsis of the Pew Environmental Health Tracking Project. Environmental Health Perspectives, 112, 1414-1418. http://dx.doi.org/10.1289/ehp.7147

[2] Jansen, M. (2007) Collaboration between Practice, Policy and Research in Local Public Health. Universiteit Maastricht, Maastricht.

[3] International Commission on Occupational Health (1993) Policy Document of the Scientific Committee on Health Services Research and Evaluation in Occupational Health. ICOH, Amsterdam.

[4] Israel, B.A., Parker, E.A., Rowe, Z., Salvatore, A., Minkler, M., Lopez, J., Butz, A., Mosley, A., et al. (2005) Community-Based Participatory Research: Lessons Learned from the Centers for Children's Environmental Health and Disease Prevention Research. Environmental Health Perspective, 113, 1463-1471. http://dx.doi.org/10.1289/ehp.7675

[5] Pope, C. and Mays, N. (1993) Opening the Black Box: An Encounter in the Corridors of Health Services Research. BMJ, 306, 315-318. http://dx.doi.org/10.1136/bmj.306.6873.315

[6] Bonham, V.L. and Nathan, V.R. (2002) Environmental Public Health Research: Engaging Communities. International Journal of Hygiene and Environmental Health, 205, 11-18. http://dx.doi.org/10.1078/1438-4639-00125

[7] Kone, A., Sullivan, M., Senturia, K.D., Chrisman, N.J., Ciske, S.J. and Krieger, J.W. (2000) Improving Collaboration between Researchers and Communities. Public Health Reports, 115, 243-248. http://dx.doi.org/10.1093/phr/115.2.243

[8] Prince, M., Patel, V., Saxena, S., Maj, M., Maselko, J., Phillips, M.R. and Rahman, A. (2007) No Health without Men- 
tal Health: A Slogan with Substance. Lancet, 370, 859-877. http://dx.doi.org/10.1016/S0140-6736(07)61238-0

[9] Lopez, A.D., Mathers, C.D., Ezzati, M., Jamison, D.T. and Murray, C.J.L. (2006) Global Burden of Disease and Risk Factors. Oxford University Press and World Bank, New York. http://dx.doi.org/10.1596/978-0-8213-6262-4

[10] WHO (2002) NCD Research Partners. Meeting Report. World Health Organization, Geneva.

[11] Bachrach, L. (2000) The Past and Future of Mental Health Services: An Interview with Leona Bachrach. Psychiatric Services, 51, 1511-1512. http://dx.doi.org/10.1176/appi.ps.51.12.1511

[12] Geller, J.L., Fisher, W.H., McDermeit, M. and Brown, J.M. (1998) The Effects of Public Managed Care on Patterns of Intensive Use of Inpatient Psychiatric Services. Psychiatric Services, 49, 327-332.

[13] Jorm, A., Griffiths, K., Christensen, H. and Medway, J. (2001) Research Priorities in Mental Health (No. 3167). Centre for Mental Health Research, Canberra.

[14] Mental Health Council of Australia and Brain and Mind Research Institute (2005) Not for Service. Canberra.

[15] Parker, G. and Parker, K. (2002) A Profile of Regional Psychiatry Publishing: Home and Away. Australian \& New Zealand Journal of Psychiatry, 36, 693-696. http://dx.doi.org/10.1046/j.1440-1614.2002.01075.x

[16] Upshur, R. (2002) If Not Evidence, Then What? Or Does Medicine Really Need a Base? Journal of Evaluation in Clinical Practice, 8, 113-119. http://dx.doi.org/10.1046/j.1365-2753.2002.00356.x

[17] Paraje, G., Sadana, R. and Karam, G. (2005) Increasing International Gaps in Health-Related Publications. Science, 308, 959-960. http://dx.doi.org/10.1126/science.1108705

[18] Mcallister, M., Munday, J., Taikato, M., Waterhouse, B. and Dunn, P.K. (2012) Determining Mental Health Research Priorities in a Queensland Region: An Inclusive and Iterative Approach with Mental Health Service Clinicians, Consumers and Carers. Advances in Mental Health, 10, 268-276. http://dx.doi.org/10.5172/jamh.2012.10.3.268

[19] Vega, W.A. and Lopez, S.R. (2001) Priority Issues in Latino Mental Health Services Research. Mental Health Services Research, 3, 189-200. http://dx.doi.org/10.1023/A:1013125030718

[20] Polit, D. (2010) Statistics and Data Analysis. Lippincott, New York.

[21] Lichtman, M. (2010) Qualitative Research in Education: A User’s Guide. 2nd Edition, Sage, California.

[22] Polit, D. and Hungler, B. (2008) Essential of Nursing Research: Methods Appraisal and Utilization. Lippincott, New York.

[23] Tomlinson, M., Rudan, I., Saxena, S., Swartz, L., Tsai, A.C. and Patel, V. (2009) Setting Priorities for Global Mental Health Research. Bulletin of the World Health Organisation, 87, 438-446. http://dx.doi.org/10.2471/BLT.08.054353

[24] Gregorio, G., Tomlinson, M., Gerolin, J., Kieling, C., Moreira, H.C., Razzouk, D. and Mari, J. (2012) Setting Priorities for Mental Health Research in Brazil. Revista Brasiieira de Psiquiatria, 34, 434-439. http://dx.doi.org/10.1016/j.rbp.2012.05.006

[25] Jorm, A., Griffiths, K., Christensen, H. and Medway, J. (2001) Research Priorities in Mental Health (No. 3167). Centre for Mental Health Research, Canberra.

[26] WHO (2011) Mental Health Atlas 2011. Department of Mental Health and Substance Abuse, Occupied Palestinian Territory, World Health Organization. www.who.int/mental_health/evidence/atlas/profiles/pse_mh_profile.pdf

[27] WHO, Department of Vaccines and Biologicals (2001) Making Surveillance Work. Module 3: Logistics Management. http://whqlibdoc.who.int/hq/2001/WHO_V\&B_01.10.pdf

[28] WHO, Regional Office for the Eastern Mediterranean (2010) Maternal, Child and Adolescent Mental Health: Challenges and Strategic Directions 2010-2015. www.emro.who.int/docs/EM_RC57_3_en.pdf

[29] Ministry of Health, PHIC (2012) Annual Health Report, Palestine 2011. 
Scientific Research Publishing (SCIRP) is one of the largest Open Access journal publishers. It is currently publishing more than 200 open access, online, peer-reviewed journals covering a wide range of academic disciplines. SCIRP serves the worldwide academic communities and contributes to the progress and application of science with its publication.

Other selected journals from SCIRP are listed as below. Submit your manuscript to us via either submit@scirp.org or Online Submission Portal.
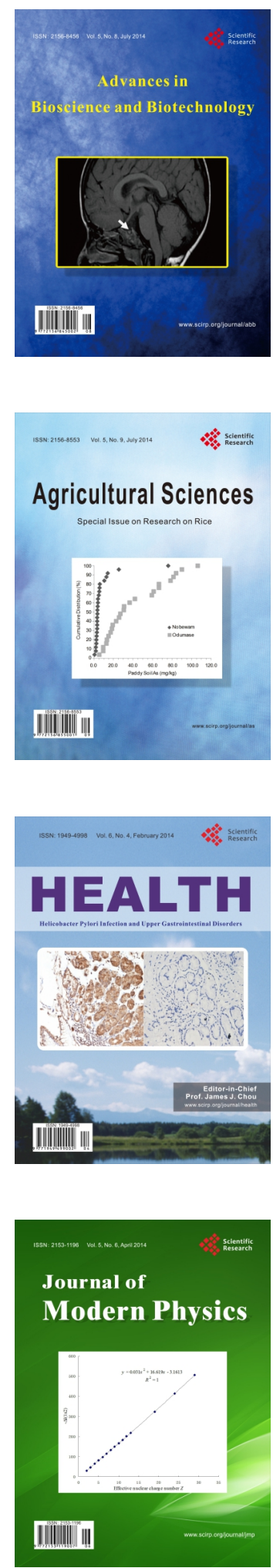
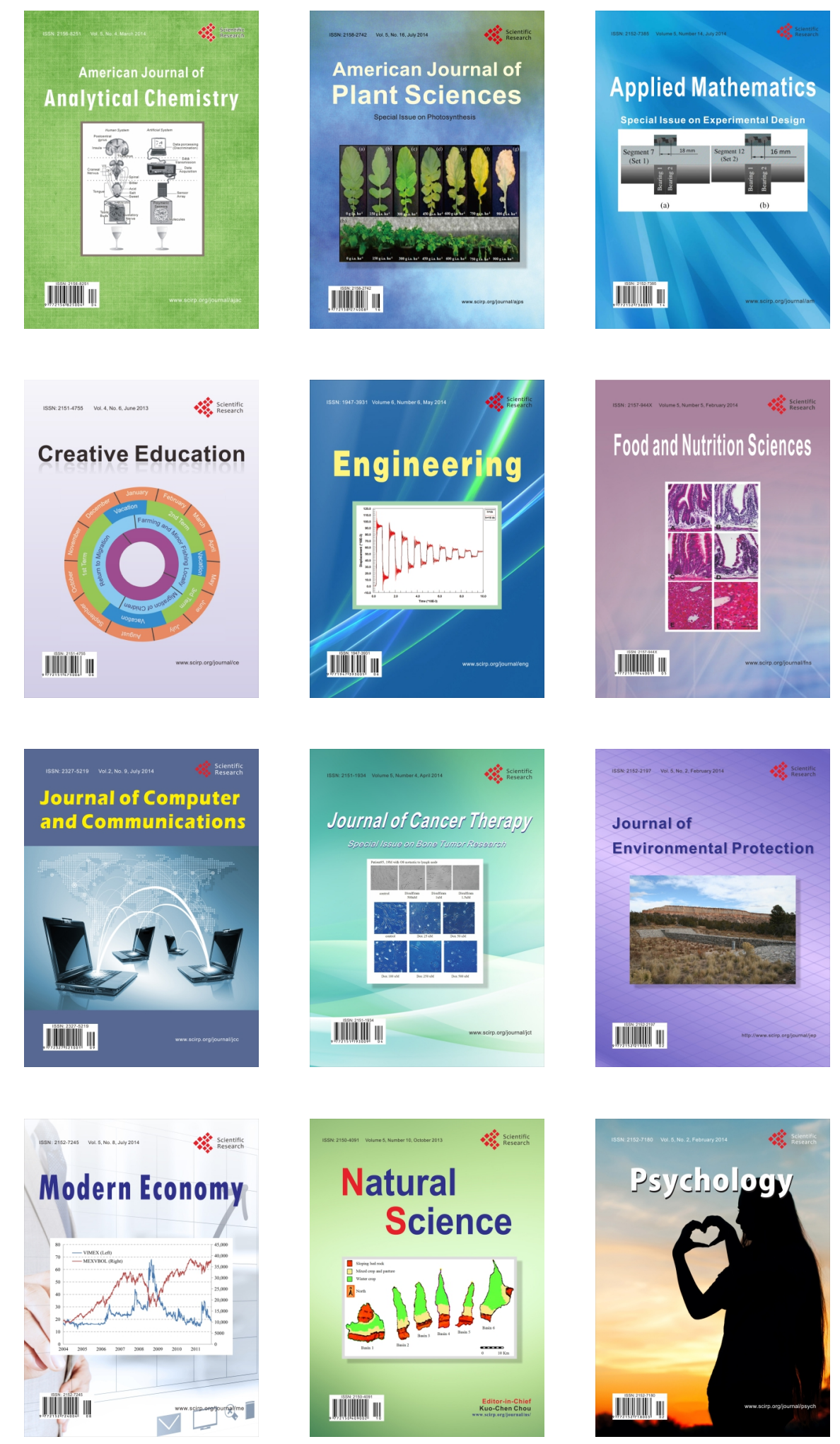